\title{
The contribution of individual psychological features to the determination of the phenomenon of work alienation in the "person - person" professions type
}

Anastasiia A. Kozhina, Department of psychology of professional activity, Herzen State Pedagogical University of Russia

Leonid V. Vinokurov, Laboratory of psychology and psychophysiology of sport, Saint-Petersburg Scientific Institute for physical culture

Work alienation is a phenomenon which means "a distance or separation from work, its context or itself" [1], represents subjectively endured state connected with destruction of interpersonal communications which is expressed in perception by the subject of work of helplessness, senselessness, self-alienation.

Specialists have found links between work alienation and decreased work efficiency, increased alcoholism and destructive behaviors [2], which indicates the high importance of this problem. Meanwhile, the question of psychological predictors of construct remains a little studied (especially in the Russian-language sample).

\section{Materials and Methods}

Using the questionnaire method, 92 employees of the "person-person" professions type were surveyed (34\% male and $66 \%$ female, age $\varnothing 41,4$ years).

- Work alienation was measured by "Questionnaire of work alienation concrete job" [3]; adapted by Vinokurov and Kozhina.

- Personality traits were studied using the Freiburger Persönlichkeitsinventar (FPI) in adaptation of Krylov and Ronginskaya [4].

- The scale of satisfaction of basic psychological needs at work in adaptation of Osin was applied [5].
Based on prior studies of the relationship of related to work alienation constructs (work involvement and job satisfaction) and personality traits $[6,7]$, it is possible to make the assumption of presence of those concerning alienation.

Table 1. Spearman correlation values of Questionnaire of work alienation concrete job and FPI scales.

\begin{tabular}{lc}
\hline & Work alienation \\
\hline FPI - depression & $.43^{* *}$ \\
FPI - sociability & $.42^{* *}$ \\
FPI - reactive & $.32^{* *}$ \\
aggressiveness & $.34^{* *}$ \\
FPI - introversion & $.38^{* *}$ \\
FPI - emotional lability & \\
$* * p<0.01$. &
\end{tabular}

In consistency with previous studies work alienation showed a moderate association with such personality traits as depression, shyness, introversion, emotional lability, reactive aggressiveness and sociability. These findings allow developing of a differentiated approach to the prevention and intervention of work alienation, depending on the personality traits of employees. Based on the fact that personality traits represent stable psychological constructs, we can conclude that in constant, stable environmental conditions, work alienation is also a relatively steady and stable over time construct.
Based on the Self-Determination-Theory [8], we assumed that the intensity of the phenomenon is determined by the level of satisfaction of basic psychological needs at work.

Table 2. Effect of satisfaction of need for autonomy on work alienation.

\begin{tabular}{lcccc} 
Predictor & $\mathrm{B}$ & $\mathrm{SE}$ & $\beta$ & $\mathrm{R}^{2}$ \\
\hline Autonomy & 0.82 & 0.72 & $0.45^{* *}$ & $-.28^{* *}$ \\
${ }^{* *} \mathrm{p}<.01$ & & & &
\end{tabular}

Table 3. Effect of satisfaction of need for competence on work alienation.

\begin{tabular}{lcccc} 
Predictor & $\mathrm{B}$ & $\mathrm{SE}$ & $\beta$ & $\mathrm{R}^{2}$ \\
\hline $\begin{array}{l}\text { Competence } \\
{ }^{* *} \mathrm{p}<.01\end{array}$ & 1.26 & 0.48 & $0.53^{* *}$ & $-.29^{* *}$
\end{tabular}

Table 4. Effect of satisfaction of need for relatedness on work alienation.

\begin{tabular}{lcccc} 
Predictor & $\mathrm{B}$ & $\mathrm{SE}$ & $\beta$ & $\mathrm{R}^{2}$ \\
\hline $\begin{array}{l}\text { Relatedness } \\
{ }^{* *} \mathrm{p}<.01\end{array}$ & 0.60 & 0.17 & $0.57^{* *}$ & $-.31^{* *}$
\end{tabular}

The results of the linear regression analysis presented in Tables 1-3 support that work alienation is negatively predicted by satisfaction for needs for autonomy, competence and relatedness.
The satisfaction of these needs is possible within conditions and the content of concrete work. Therefore, depending on these factors, alienation from work can be considered as rather mobile construct. Thus, an organization should offer working conditions that allow employees to act self-determined, to foster need satisfaction and therefore prevent work alienation.

\section{Practical implications}

Regarding practical implications, our results mean new positive prospects for employers as they lead to the assumption that alienation can be altered by changes in the working environment. This opens space for measurements towards employee's satisfaction as for example job enlargement, job enrichment or the implementation of transactional leadership.

\section{Distribution of employment areas in the sample}

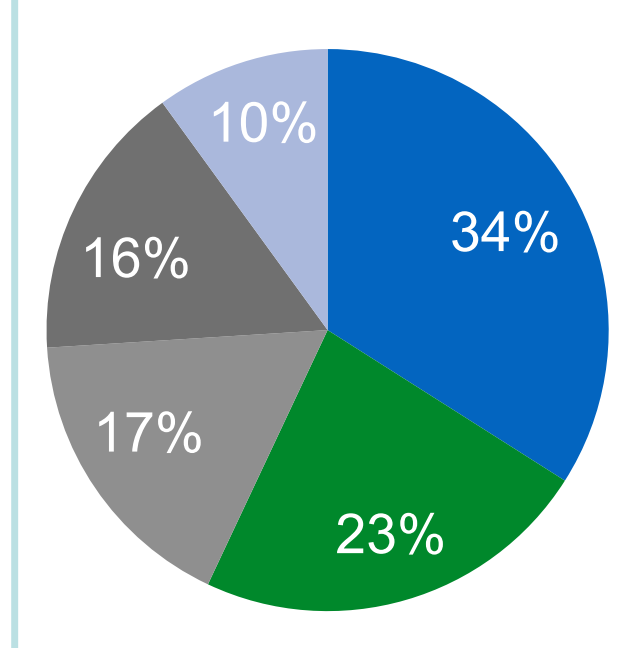

- education

management

medicine

- psychology

other 